\title{
Keine Unterschiede im Überleben und bei der Lebensqualität?
}

Hintergrund und Fragestellung: Reicht die Bestrahlung des Glioblastoms über eine Woche aus? Die Strahlentherapie der erweiterten Tumorregion ist Standard in der Behandlung des Glioblastoms.

Bei der Wahl des genauen Fraktionierungsschemas spielen prognostische Faktoren eine Rolle: Je schlechter die Lebenserwartung ist, desto weniger relevant sind neurokognitive Spätfolgen aufgrund der Strahlentherapie. Dies führte vor einigen Jahren zur Etablierung einer akzelerierten Bestrahlung von 40 Gy in 15 Fraktionen über drei Wochen, die einer konventionellen Bestrahlung in Fraktionen von 30 x 2 Gy über sechs Wochen nicht unterlegen war.

In einer weiteren Entwicklung dieser Behandlungsphilosophie wurde dieses akzelerierte Protokoll nun mit einer deutlich gestrafften Strahlentherapie, die sich nur über eine Woche erstreckte, verglichen.

\section{Originalie}

Roa W et al. International Atomic Energy Agency randomized phase III study of radiation therapy in elderly and/or frail patients with newly diagnosed glioblastoma multiforme. J Clin Oncol. 2015;33(35):4145-50.
Patienten und Methodik: Zwischen 2010 und 2013 wurden 98 Glioblastom-Patienten in 12 Zentren 1:1 randomisiert einer postoperativen Radiotherapie (RT) zugeführt: entweder mit 5 x 5 Gy über eine Woche (Kurzzeit-RT) oder mit 15 Fraktionen über drei Wochen bis zu einer
Gesamtdosis von 40 Gy (Standard-RT). Eingeschlossen wurden Patienten, die entweder 50 Jahre alt waren und einen KarnofskyPerformancestatus (KPS) von 50 - 70\% hatten oder aber 65 Jahre oder älter waren und einen KPS von $50-70 \%$ hatten oder 65 Jahre oder älter waren und einen KPS von $80-100 \%$ hatten.

Ergebnisse: Die akzelerierte Strahlentherapie über eine Woche war der Vergleichsgruppe nicht unterlegen: das mediane Überleben betrug 7,9 Monate (95\%-Konfidenzintervall [95\%-KI], 6,3-9,6 Monate) gegenüber 6,4 Monaten (95\%-KI, 5,1-7,6 Monate) $(\mathrm{p}=0,988)$. Das mediane progressionsfreie Überleben betrug 4,2 Monate in beiden Armen. Bei einer medianen Nachbeobachtungszeit von 6,3 Monaten war die Lebensqualität, gemessen anhand der Lebensqualitätsfragebögen QLQ-C30 und QLQBN20 der European Organisation for Research and Treatment of Cancer (EORTC) zu Beginn sowie nach vier und nach acht Wochen nicht unterschiedlich.

Schlussfolgerungen der Autoren: Die einwöchige Strahlentherapie könne als Behandlungsoption für ältere oder "gebrechliche" (frail) Patienten mit neu diagnostizierten Glioblastomen empfohlen werden, weil es keine Unterschiede im Gesamtüberleben, progressionsfreien Überleben oder bezüglich Lebensqualität gebe.

Schlüsselwörter: Glioblastom - Radiotherapie-Regime - ältere Patienten - fragile Patienten

\section{- Kommentar von Michael Weller, Zürich, Schweiz}

\section{„Diese Studie sollte die Therapiestandards in Deutschland nicht wesentlich beeinflussen"}

Es liegt eine pragmatische randomisierte Studie vor, die potenziell einen neuen Standard in der Therapie des Glioblastoms bei älteren oder gebrechlichen Patienten für Länder definiert, die über begrenzte Ressourcen im Gesundheitssystem verfügen. Bezüglich der Wirksamkeit der Strahlentherapie sind die Ergebnisse nachvollziehbar, bezüglich der "Lebensqualitätserhebung" ist festzuhalten, dass bereits nach acht Wochen nur noch für die Hälfte der Patienten solche Daten vorlagen und dass diesbezüglich, im Gegensatz zur Darstellung der Autoren, keine vernünftige Aussage gemacht werden kann.

In einer sich rasch entwickelnden Onkologie, in der zunehmend basierend auf molekularen Markern behandelt wird speziell im Falle des Glioblastoms bei älteren Patienten, basierend auf dem MGMT-Promotor-Methylierungsstatus - wirkt diese Studie eher wie ein Schritt in die Vergangenheit, und es ist kaum vorstellbar, dass ein Bestrahlungsregime mit 5 x 5 Gy keine negativen Auswirkungen auf die Hirnfunktion bei älteren Patienten haben wird, wenn aufgrund weiterer Fortschritte in der medikamentösen Therapie längere Überlebenszeiten erzielt werden. Die Ergebnisse dieser Studie sollten die Therapiestandards in Deutschland eher nicht wesentlich beeinflussen.

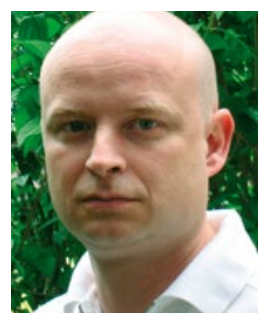

Prof. Dr. Michael Weller, Zürich, Schweiz

Klinik für Neurologie, Universitätsspital Zürich michael.weller@usz.ch 